\title{
The Origin of X-ray Emission from the Enigmatic Be Star $\gamma$ Cassiopeiae
}

\author{
K. Hamaguchi ${ }^{1,2}$, L. Oskinova ${ }^{3}$, C. M. P. Russell ${ }^{4}$, R. Petre $^{4}$, \\ T. Enoto ${ }^{5,6}$, K. Morihana ${ }^{7}$ and M. Ishida ${ }^{8}$
}

${ }^{1}$ CRESST II and X-ray Astrophysics Laboratory NASA/GSFC, Greenbelt, MD 20771, USA: Kenji.Hamaguchi@nasa.gov, ${ }^{2}$ Department of Physics, University of Maryland, Baltimore County, 1000 Hilltop Circle, Baltimore, MD 21250, USA, ${ }^{3}$ Institute of Physics and Astronomy, University of Potsdam, 14476 Potsdam, Germany, ${ }^{4} \mathrm{X}$-ray Astrophysics Laboratory NASA/GSFC, Greenbelt, MD 20771, USA, ${ }^{5}$ The Hakubi Center for Advanced Research, Kyoto University, Kyoto 606-8302, Japan, ${ }^{6}$ Department of Astronomy, Kyoto University, Kitashirakawa- Oiwake-cho, Sakyo-ku, Kyoto 606-8502, Japan, ${ }^{7}$ Nishi-Harima Astronomical Observatory, Center for Astronomy, University of Hyogo, 407-2, Nichigaichi, Sayo-cho, Sayo, Hyogo, 670-5313, Japan, ${ }^{8}$ The Institute of Space and Astronautical Science, Japan Aerospace Exploration Agency, 3-1-1 Yoshinodai, Chuo-ku, Sagamihara, 252-5210, Japan

\begin{abstract}
Gamma Cassiopeiae is an enigmatic Be star with unusually hard, strong X-ray emission compared with normal main-sequence B stars. The origin has been debated for decades between two theories: mass accretion onto a hidden compact companion and a magnetic dynamo driven by the star-Be disk differential rotation. There has been no decisive signature found that supports either theory, such as a pulse in X-ray emission or the presence of large-scale magnetic field. In a $\sim 100$ ksec duration observation of the star with the Suzaku X-ray observatory in 2011, we detected six rapid X-ray spectral hardening events called "softness dips". All the softness dip events show symmetric softness ratio variations, and some of them have flat bottoms apparently due to saturation. The softness dip spectra are best described by either $\sim 40 \%$ or $\sim 70 \%$ partial covering absorption to $k T \sim 12 \mathrm{keV}$ plasma emission by matter with a neutral hydrogen column density of $\sim 2-8 \times 10^{21} \mathrm{~cm}^{-2}$, while the spectrum outside of these dips is almost free of absorption. This result suggests that two distinct X-ray emitting spots in the $\gamma$ Cas system, perhaps on a white dwarf companion with dipole mass accretion, are occulted by blobs in the Be stellar wind, the Be disk, or rotating around the white dwarf companion. The formation of a Be star and white dwarf binary system requires mass transfer between two stars; $\gamma$ Cas may have experienced such activity in the past.
\end{abstract}

Keywords. stars: emission-line, Be — stars: individual ( $\gamma$ Cassiopeiae $)$ - stars: winds, outflows - X-rays: stars — white dwarfs — blue stragglers

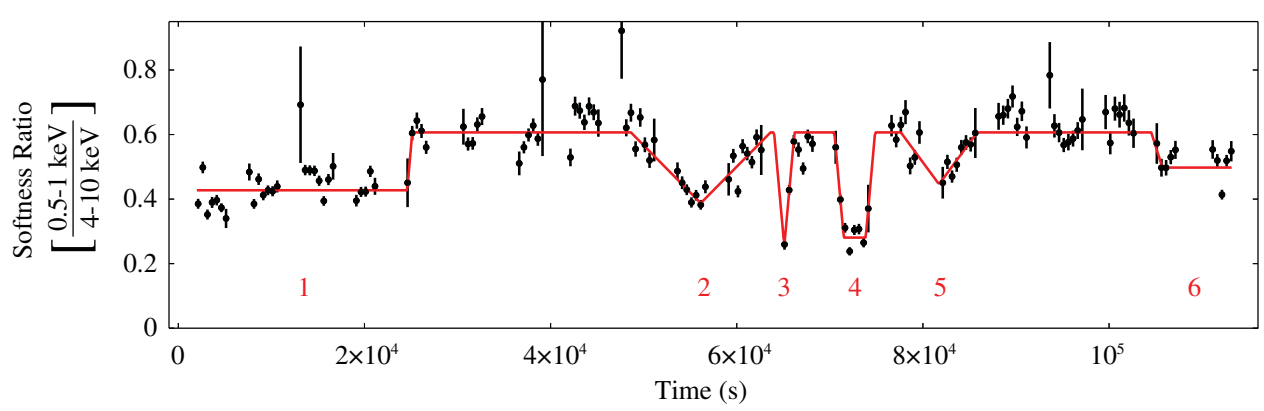

Figure 1. Time series of a softness ratio - the $0.5-1 \mathrm{keV}$ count rate over the $4-10 \mathrm{keV}$ count rate - measured with the Suzaku X-ray observatory in 2011. The solid line and numbers in red show the best-fit of an empirical softness dip model and the detected six softness dips. See Hamaguchi et al. 2016, Astrophysical Journal, 832, 140 for details. 